\title{
Scanning Scattering Contrast Microscopy for Actinic EUV Mask Inspection
}

\author{
I. Mohacsi*a, P. Helfenstein ${ }^{\mathrm{a}}$, R. Rajendran ${ }^{\mathrm{a}}$, and Y. Ekinci ${ }^{\mathrm{a}}$ \\ ${ }^{a}$ Paul Scherrer Institute, Villigen PSI, CH-5232, Switzerland \\ *istvan.mohacsi@psi.ch
}

\begin{abstract}
Actinic mask inspection for EUV lithography with targeted specification of sensitivity and throughput is a big challenge and effective solutions are needed. We present a novel method for actinic mask inspection, i.e. scanning scattering contrast microscopy. In this method the EUV mask is scanned with a beam of relatively small spot size and the scattered light is recorded with a pixel detector. Since the mask layout is known, the scattering profile of a defect-free mask at the detector can be calculated. The signal between the measured and calculated signal provides the deviation between the real mask and its ideal counterpart and a signal above a certain threshold indicates the existence of a defect within the illumination area. Dynamic software filtering helps to suppress strong diffraction from defect free structures and allows registration of faint defects with high sensitivity. With the continuous scan of the whole mask area, a defect map can be obtained with high throughput. Therefore, we believe that this method has the potential of providing an effective solution for actinic mask inspection. Here we discuss the basic principles of the method, present proof-of-principle experiments, describe the basic components of a feasible stand-alone tool and present early results of the performance estimations of such a tool.
\end{abstract}

Keywords: EUV lithography, actinic inspection, lensless imaging, mask metrology, defect characterization

\section{INTRODUCTION}

One of the major challenges of EUV lithography is actinic mask inspection. The true nature and printability of the defects can be fully validated by optical metrology at the illumination angle of 6 degrees and $13.5 \mathrm{~nm}$ wavelength. Therefore actinic metrology is an indispensable part of EUV mask infrastructure [1]. Actinic metrology is needed for blank inspection, patterned mask inspection, mask review and final (thru pellicle) inspection. Tools for mask review and blank mask inspection are in progress [2,3]. However, in particular patterned mask and final inspection still remain as a major challenge. Effective solutions with targeted specifications of sensitivity, throughput, and resolution as well as with high availability, small footprint, and low cost of ownership and maintenance are on high demand for this metrology gap.

Here we propose a solution to this challenge. We present a novel method for actinic mask inspection, i.e. scanning scattering contrast microscopy. We discuss the basic principles of the method, present proof-of-principle experiments, describe the basic components of a feasible stand-alone tool, and present early results of the performance estimations of such a tool.

\section{LENSLESS METROLOGY}

Conventional optical microscopy employs a series of lenses in its optical setup, where the field-of-view on the sample is uniformly illuminated and scatters the incoming light according to its spatial frequencies. The pass through the objective collects part of the angular spectrum and the optics effectively perform (inverse) Fourier transforms to produce a magnified aerial image of the sample at the image plane. Spatial filtering is an effective and well-established method to increase the sensitivity in optical inspection methods. A spatial filter can be easily placed at an aperture plane (i.e. Fourier space) of the microscope, and can be designed to absorb the strong signals coming from the designed mask layout to detect the low signals due to defects. Such spatial filters correspond to the Fourier transform of the ideal pattern and are either pre-manufactured absorber plates or programmable liquid crystal filters. For example, dark-field imaging

Metrology, Inspection, and Process Control for Microlithography XXX, edited by Martha I. Sanchez, Vladimir A. Ukrainstev Proc. of SPIE Vol. 9778, 977810 - (c) 2016 SPIE · CCC code: 0277-786X/16/\$18 · doi: 10.1117/12.2220027 
is the simplest form of spatial filtering in which the low spatial frequencies of the illumination function are blocked by the collection optics in order to see the small defects on a highly reflecting EUV mask blank $[2,4,5,6]$.

For EUV patterned mask inspection, which requires extremely high throughput and sensitivity, there has been continuous and forward-looking progress. Nevertheless, the availability of effective actinic mask inspection tools has been bootstrapped with several issues. The major challenge turned out to be EUV sources with high brightness, stability, and availability and low maintenance. Moreover, EUV inspection needs a detector with high dynamic range, since the quantum yield of a single EUV photon is about 25 electrons. In addition, the complexity of the systems and optics are also challenging. The progress in these technologies is ongoing and there is a significant potential of improvement.

While the developments in conventional microscopy techniques saturated over the last century, lensless microscopy techniques have emerged as a field of intense research and current progress [7-12]. A comparison between the lens-based (real space) and lensless (reciprocal space) microscopy is illustrated in Fig. 1. In general, these methods rely on the fact that the intensity profile at the detector plane corresponds to the Fourier domain (reciprocal space) of the sample. However, as the detector records only the intensity of the incoming radiation, the phase information gets lost during the detection process. This so-called phase problem prevents the use of a single Fourier transform back projection to recover the aerial image at the sample plane. Therefore, lensless microscopy techniques employ various computational algorithms and real and reciprocal space constraints to recover the phase and reconstruct the aerial image of the sample

Lensless microscopy bears several appealing properties over conventional microscopy, including: (1) Reciprocal space imaging does not require any imaging optics, leading to a greatly simplified optical setup. (2) The resolution is provided by the solid angle covered by the detector and therefore can be easily tuned by the placement of the detector or by changing the size of the one single component - the detector. (3) Diffractive imaging does not have a strict focal depth limitation, given that condenser-to-sample and sample-to-detector distances are relatively long. (4) Since the aforementioned distances are long, imaging through pellicle is possible. (5) Since, the reconstruction algorithms naturally retrieves the phase, the reciprocal techniques provide inherent phase contrast maps, which is crucial in identifying phase defects.

On the other hand, it also has certain disadvantages: (1) It requires a source of much higher brightness compared to fullfield microscopy, since it relies on the coherence properties of the light. (2) Since the interpretation of diffraction patterns is not straightforward, the iterative algorithms employed for aerial image recovery are more demanding in computational power. (3) Existing algorithms generally require high data quality.

Owing to its obvious advantages compared to real-space imaging, several research groups studied the use of scatterometry [13], CDI [14] or ptychography [15,16] for actinic mask metrology. This can also be observed from the present conference, where a considerable number of contributions explored the possibility of coherent diffraction for mask metrology. Experimental techniques like CD-SAXS [17] have been successfully employed for the measurement of roughness or CD uniformity. Reciprocal space imaging techniques, like coherent diffractive imaging (CDI) [13], ptychography $[15,18]$ or Fourier ptychography [19] have been successfully demonstrated by several groups. Along with other research groups, we made significant progress in lensless EUV imaging [16], yet it needs substantial further development to address all issues required to meet the strict target specifications of EUV mask inspection. This prompted us to look for out-of-the-box solutions using the additional information that the masks layouts are given and thereby the aerial image of the sample can be calculated. Coupling this a-priori knowledge into the defect inspection system can substantially simplify the lensless imaging methods and boost their performance. There are several ways of making use of this a-priori information.

Here, we propose a novel, high throughput method for lensless actinic mask inspection, i.e. scanning scattering contrast microscopy (SSCM). The proposed method is analogous to scanning small angle X-ray scattering (scanning SAXS) and exploits the advantage of a-priori knowledge of the expected absorber pattern. In this method EUV mask is scanned with a beam of relatively small spot size (of the order of $10 \mathrm{~s}$ of microns) and its diffraction patterns are recorded with a pixel detector (as shown in Fig. 1). Since the mask layout is known, the diffraction patterns of a defect free mask can be calculated through exact, non-iterative algorithms. By calculating the difference between the measured and calculated signal, one can obtain a map of deviations between the real and ideal mask. When the difference is, above a certain (adaptive) threshold, it indicates the presence of a defect within the illumination area. In addition to calculated patterns, die-to-database and die-to-die approaches are also feasible because of the low density of the defects compared to the whole area of the mask. 
Since mask structures are often relatively regular repeating structures within a limited range of size and shapes (compared to samples from nature), they have a limited range of spatial frequencies. Therefore the diffraction pattern from many mask structures exhibit strong peaks while defect scattering can be detected as a faint signal in the background. Figure 2 shows a detector image of a typical memory device, revealing strong diffraction peaks. The signal from a small defect is only visible when the intensity is scaled up. The signal to noise ratio can be substantially increased when the strong peaks are not taken into account in the scattering contrast signal. As the measurement plane is the Fourier domain, the spatial frequency filtering is straightforward in SSCM by applying software filters on them. Moreover, using software methods, several filters can be defined on the same diffraction pattern to obtain different contrast mechanisms. For example in Fig. 2, the saturated peaks cannot be measured anyway, but two complementary filters can be employed on the rest of the measured patterns. One filter can be looking at areas between the peaks, where no signal is expected from perfect structures. The second filter can be monitoring the intensity difference of the nonsaturated peaks. These two contrast mechanisms should work complementary to each other, with the second mechanism better suited for complex, non-periodic patterns and the first one aimed at high periodicities. Beyond the mentioned two, several other filters can also be applied to other parts of the detector to improve sensitivity.

With the continuous scan of the whole mask area and calculating the differences with a particular filter, a defect map can be obtained with high throughput. The position accuracy of a detected defect is limited to the spot size and overlap factor of spots, and is, therefore, limited (e.g. to several microns). After high-throughput localization of the defect sites, the defects can be localized more accurately and characterized in detail by either performing a slow SSCM (e.g. small spot and more overlap) or by reconstructing the aerial image using scanning coherent diffraction imaging (SCDI) methods with the same tool.
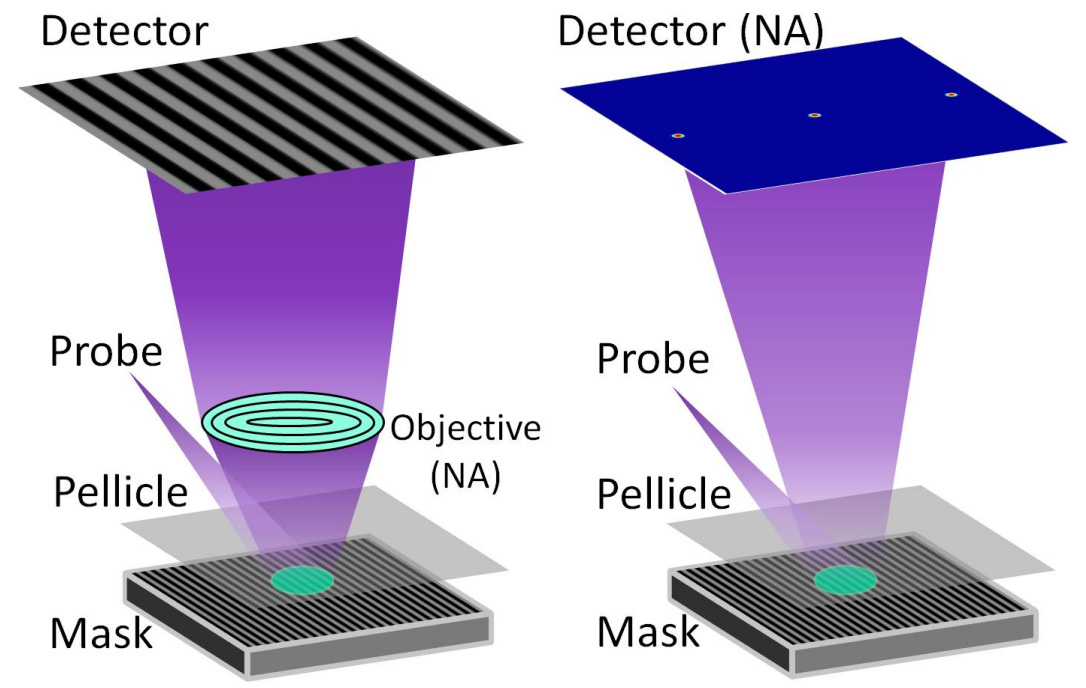

Figure 1: Comparison between the lens-based (real space) and lensless (reciprocal space) microscopy. In real space imaging (left), the resolution is given by the NA of the objective which must be placed close to the mask surface, making the system spatially constrained over the pellicle. In reciprocal space imaging (right), resolution is rather given by the NA of the detector, which due to its larger rectangular layout offers more space over the mask surface, relaxing the constraints on design and in pellicle management. 

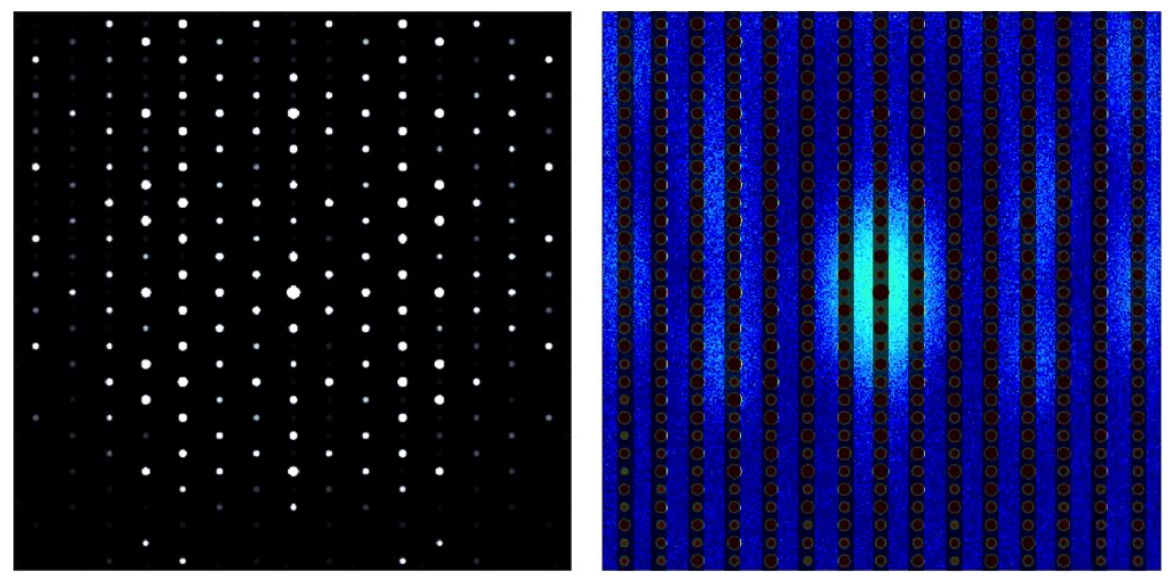

Figure 2: Left: Simulated diffraction pattern of an SRAM cell contact hole array on a detector, showing sharp, often saturated diffraction peaks. Right: Due to large intensity differences in reciprocal space, the same image is plotted on logarithmic scale, revealing the diffuse scattering present in the background. By masking out the peaks coming from the structure, we can simply sum up all intensity outside the patterns to obtain the "defectivity" of the structures at the particular scan position.

\section{EXPERIMENTAL RESULTS}

To demonstrate the proof-of-principle of SSCM, we performed experiments at the XIL-II beamline of the Swiss Light Source (SLS) using the RESCAN 1+ setup [11]. The XIL beamline provides spatially coherent EUV light at $13.5 \mathrm{~nm}$ wavelength with a bandwidth of $4 \%$. The incoming light was focused onto a pinhole (12 $\mu \mathrm{m}$ diameter) using a Fresnel zone plate, leading to an illumination spot on the sample with $18 \mu \mathrm{m}$ diameter, incident angle of $6^{\circ}$ and $0.5 \%$ bandwidth (We note that for SSCM the bandwidth is, in general, not a critical issue). The light reflected from the mask surface was coupled out through a larger exit window of $300 \times 300 \mu \mathrm{m}^{2}$. The diffraction patterns were recorded on a CCD detector (PI MTE 2048B) at a distance of $\sim 77 \mathrm{~mm}$ over the sample plane, allowing the capture of spatial frequencies down to $\sim 50 \mathrm{~nm}$. The concept was tested on Mo/Si multilayer substrates patterned with HSQ absorber gratings with $100 \mathrm{~nm}$ half pitch (on mask). The grating was pre-patterned with periodic defects of various sizes. An optical microscope preview of the sample also revealed the presence of randomly positioned additional particles on the surface from contamination. The largest pre-patterned defects were CD errors of $\pm 40 \times 2000 \mathrm{~nm}$ (on mask dimensions). The sample was scanned in two dimensions with a piezo driven stage in closed loop over an area of $100 \times 100 \mu \mathrm{m}^{2}$. At each scan point the sample was exposed for $200 \mathrm{~ms}$ for capturing the diffraction patterns.

Figure 3 shows examples of typical measured diffraction patterns from defective and a non-defective scan positions. While the patterns show some ghost images and artifacts, the expected three main peaks (i.e. zeroth and first order diffraction peaks) of the absorber pattern can be easily recognized as oversaturated stripes subjected to blooming. When a defect enters the spot the intensity scattered between the peaks drastically increases, as a clear sign of a breach of the otherwise perfectly periodic layout. By plotting the intensity scattered outside the peaks over the $100 \times 100 \mu \mathrm{m}^{2}$ scan area, we obtain the defect map of the scanned mask area. The sensitivity towards defect identification can be further enhanced by limiting the spatial filter down to regions of interest between the peaks. Such a defect map can be seen in Fig. 4, that not only shows the presence and distribution of defects, but after some rescaling (Fig 4.b), also the statistical relevance of the recorded features. The defect map is dominated by the presence of a large added particle (white circle). We clearly identify three programmed CD errors features (red circle) with signal to noise ratios in the range of 15-30. The defect map also provides evidence of smaller defects within the scan area (yellow circle), that are most likely smaller extrinsically added particles on the sample. 

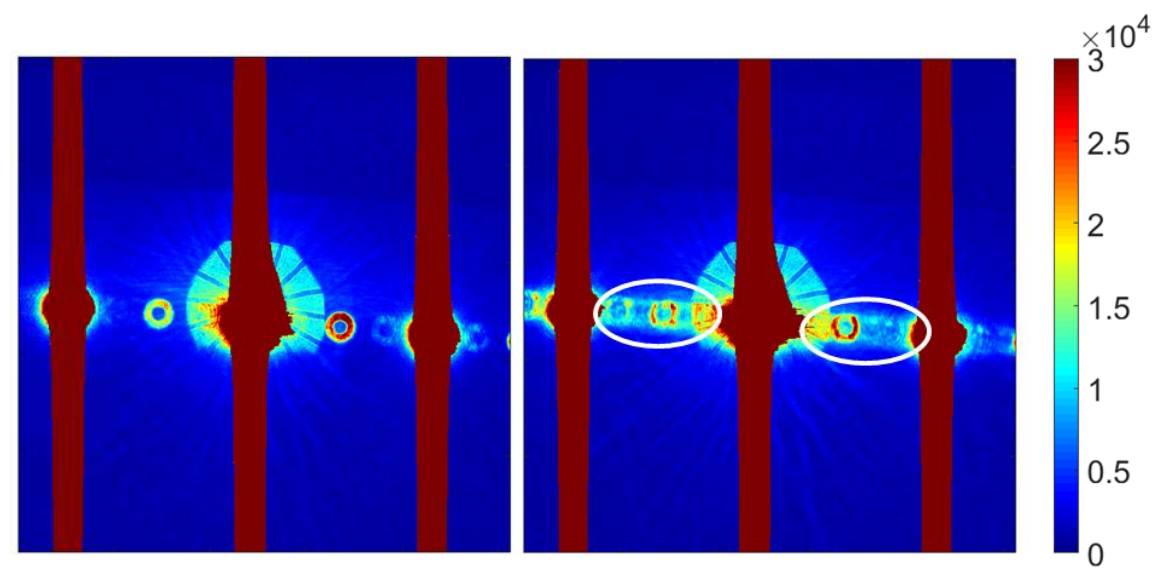

Figure 3: Examples of measured diffraction patterns without (left) and with (right) defects. The marooish patch in both the images correspond to detector blooming due to saturation of the main diffraction peaks. The marked areas highlight the defect scattering between the peaks. Besides the defect scattering, the patterns show ghost images of the fresnal zone plate, pinhole diffraction and detector blooming.
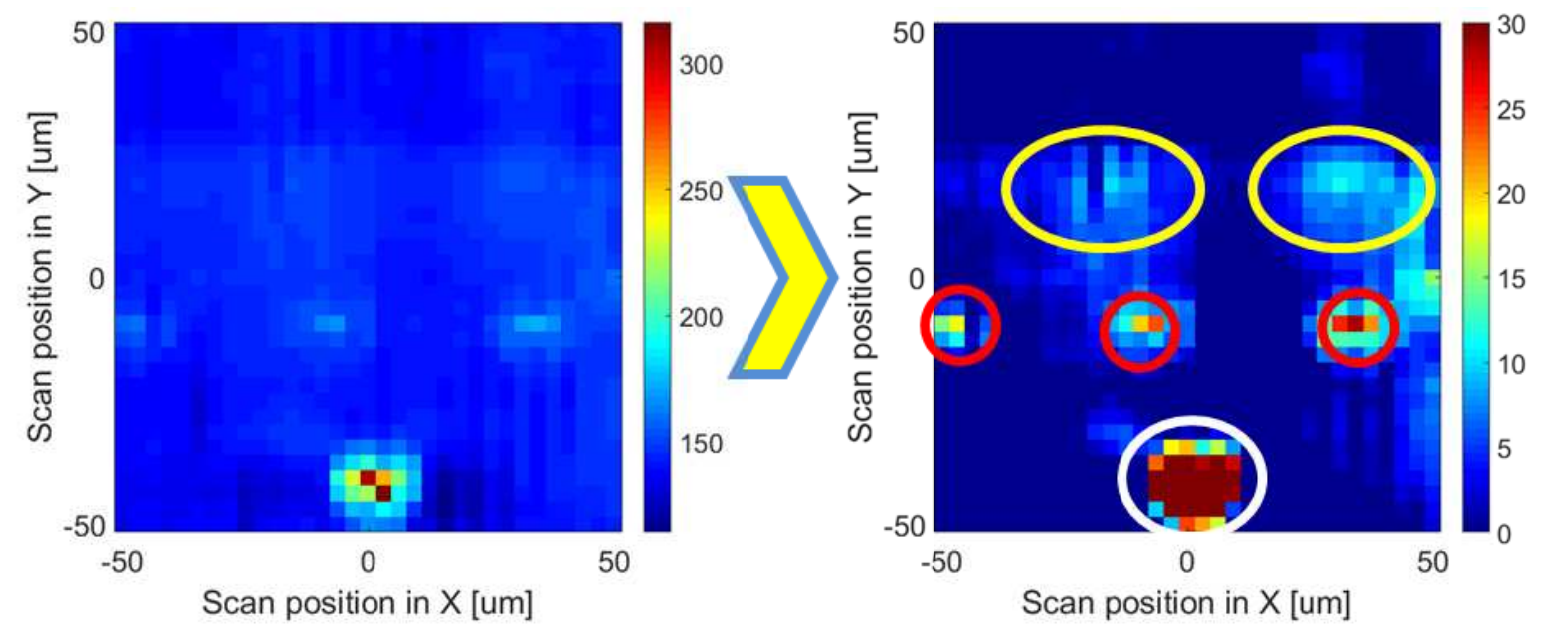

Figure 4: Defect map of a EUV mask with defects. Left and right pictures are the same scattering contrast intensity maps with different intensity scaling. The defect map reveals the presence of a large added particle (white circle) and several smaller particles (yellow circles) in addition to the programmed defects (red circles), i.e. CD errors of $10(\times 4) \mathrm{nm}$ spanning over $500(\times 4) \mathrm{nm}$. The programmed defects are detected with signal to noise ratios of more than 15 .

As seen on the above images, the measurement could clearly identify major defects with high statistical significance. Unfortunately we were unable to record smaller defects on the mask, due to the blooming of the detector even in the horizontal direction. Therefore, while the size of the recorded defects is large and they should present no significant challenge to the method itself, the achieved high signal to noise ratio for defect location proves the viability of the above presented concept but indicates the need for a dedicated setup. Such a setup would use mirrors instead of a zone plate for focusing and would be equipped by an inherently blooming free, low-noise and high framerate pixel detector.

\section{TOWARDS A STAND-ALONE ACTINIC MASK INSPECTION TOOL}

We believe that the method described above is sound and we are encouraged with the early results, and therefore we aim for developing a research tool with access to the community at the XIL-II beamline at SLS after some upgrades. However, it is of great interest for EUV community if such a method is feasible as a stand-alone tool. We would like to address feasibility of such a tool employing the methods of SCDI and SSCM and with capabilities of the targeted specs 
for the technology nodes after the HVM introduction of EUVL. For our current purpose we defined the following specifications, which may not necessarily reflect the industrial specifications according to the ITRS roadmap aiming for scanning a full mask within 6 hours with signal to noise ratio of 8.5 for printable defects.

\subsection{Chamber}

A vacuum chamber incorporating a high-precision stage, the optics, and the detector is the essential part of the system. In addition, mask handling and transfer units is to be integrated. The optical setup for lensless imaging methods is relatively simple and low cost. A condenser can focus the beam to spot size of a few microns and the desired spot size can be easily achieved by defocusing.

\subsection{The detector}

High throughput mask metrology requires a suitable detector. The detector is required to have high frame rates, low noise, high quantum efficiency and must be free of blooming. While several detector projects have been evaluated, based on performance predictions and time to market estimations, in the following sections we will concentrate our attention to hybrid pixel CMOS detectors. Hybrid pixel detectors have been originally developed for particle physics, comprising of separate collector and readout modules. The Jungfrau detector [20] represents the most recent development from PSI's detector group with 75 micron pixel size, over $2 \mathrm{kHz}$ frame rate, automatic gain switching, $50 \mathrm{e}^{-} \mathrm{rms}$ noise in high gain mode and approximately $10^{6}$ photon dynamic range at $13.5 \mathrm{~nm}$. The detector is inherently free of blooming and prototypes have already been tested for hard X-ray applications. Using a thinner entrance window, its soft X-ray version is expected to provide $60 \%$ quantum efficiency at $13.5 \mathrm{~nm}$, which can be further improved by dedicated EUV releases. Shipped in $512 \times 1024$ pixel modules, it provides an appealing option for the required high frame rate detector. A single module of this detector is used for the performance estimations in the next section, although multi-modules maybe also advantageous and further improvement of the technology is feasible. Alternatively other detector technologies can be also considered if they provide better performance.

\subsection{The source}

The right choice of the source is crucial for the proposed actinic mask inspection setup. For high throughput inspection seen in the following sections, the source should be able to reliably provide $\sim 10 \mathrm{~mW}$ coherent flux on mask, implying a $\sim 100 \mathrm{~mW}$ total output power and a brightness with more than $50 \mathrm{~kW} / \mathrm{mm}^{2} / \mathrm{sr}$. While laser induced plasma and Z-pinch sources can provide the required output power, they do not have the sufficient brightness due to the very large emittance. On the other hand while HHG sources [21] have very low emittance but cannot provide sufficient power output. Although significant progress has been made and the outlook is encouraging, in developing both plasma and HHG sources, there is still need for further development in particular for stability, availability debris mitigation and power output [22].

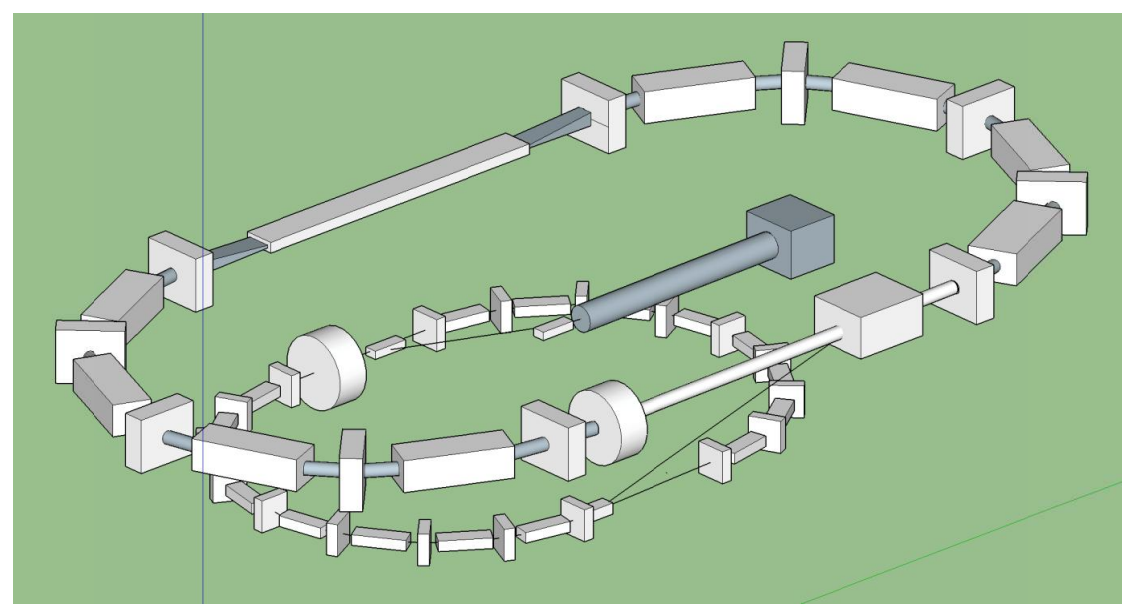

Figure 5: Stereoscopic view of the high-brightness EUV source. Electron bunches are injected from the linac into the booster ring, and after acquiring sufficient energy, they are moved to the storage ring. The storage ring is equipped with an undulator source and a conventional RF cavity. The source operates in top-up mode to provide a constant power output. 
Table 1: COSAMI electron beam and undulator parameters

\begin{tabular}{|l|c|c|}
\hline \multicolumn{1}{|c|}{ Parameter } & Symbol & Value \\
\hline \multicolumn{2}{|c|}{ Beam and lattice parameters } \\
\hline Beam energy & $E[\mathrm{MeV}]$ & 430 \\
\hline Beam current & $I[\mathrm{~mA}]$ & 150 \\
\hline Horizontal emittance & $\varepsilon_{x}[\mathrm{~nm}-\mathrm{rad}]$ & 232 \\
\hline Emittance coupling & $\kappa[\%]$ & 10 \\
\hline Relative rms energy spread & $\sigma_{E} / E$ & $3.3 \times 10^{-4}$ \\
\hline H-Beta value at U-center & $\beta_{x}[\mathrm{~m}]$ & 4.5 \\
\hline V-Beta value at U-center & $\beta_{y}[\mathrm{~m}]$ & 2.5 \\
\hline & & \\
\hline Resonance wavelength & $\lambda[\mathrm{nm}]$ & 13.5 \\
\hline Length & $L[\mathrm{~cm}]$ & 288 \\
\hline Period length & $\lambda_{U}[\mathrm{~mm}]$ & 16 \\
\hline Number of periods & $N$ & 180 \\
\hline Gap & $g[\mathrm{~mm}]$ & 8 \\
\hline K value & $K$ & 0.63 \\
\hline Magnetic field & $B[\mathrm{~T}]$ & 0.42 \\
\hline
\end{tabular}

Table 2: Photon beam characteristics

\begin{tabular}{|l|c|c|}
\hline \multicolumn{1}{|c|}{ Parameter } & Symbol & Value \\
\hline Photon flux & $\dot{N}[\mathrm{ph} / \mathrm{s} / 0.1 \% \mathrm{BW}]$ & $1,17 \times 10^{15}$ \\
\hline Brilliance & $B\left[\mathrm{ph} / \mathrm{s} / \mathrm{mm}^{2} / \mathrm{mrad}^{2} / 0.1 \% \mathrm{BW}\right]$ & $3,24 \times 10^{16}$ \\
\hline Brilliance & $B\left[\mathrm{~kW} / \mathrm{s} / \mathrm{mm}^{2} / \mathrm{sr} / 0.1 \% \mathrm{BW}\right]$ & 210 \\
\hline Horizontal beam size at the U center & $\sigma_{x}[\mathrm{~mm}]$ & 1.00 \\
\hline Vertical beam size at the U center & $\sigma_{y}[\mathrm{~mm}]$ & 0.25 \\
\hline Horizontal beam divergence at the U center & $\sigma_{x}^{\prime}[\mathrm{mrad}]$ & 0.23 \\
\hline Vertical beam divergence at the U center & $\sigma_{y}^{\prime}[\mathrm{mrad}]$ & 0.10 \\
\hline
\end{tabular}

Compact sources based on accelerator technologies have been developed in the early 1980's and 90's for X-ray lithography. For EUVL they are not feasible because the maximum flux is limited to about $10 \mathrm{~W}$ [23]. Nevertheless the requirements for metrology sources are significantly lower than that for lithography. Therefore, we performed some conceptual studies by the accelerator group at the Swiss Light Source, which lead to the COSAMI (Compact Source for Actinic Mask Inspection) project. This is designed to be in line of the needed specifications for actinic mask inspection while occupying the smallest possible footprint and relying only on well-established technologies and concepts. It has $5 \times 10 \mathrm{~m}$ footprint, in which linac, booster ring and storage ring are integrated in a compact manner as shown in Fig. 5 . Some of its important parameters are provided in Tables 1 and 2. We note that these specifications are subject to change and evaluation of different options and further conceptual studies on the source design are in progress.

\section{PERFORMANCE ESTIMATION OF A STAND-ALONE TOOL}

In this section, we will briefly discuss the initial performance estimation studies of the tool using simulations. In order to evaluate the expected sensitivity, the throughput has been fixed by setting the probing spot diameter to $40 \mu \mathrm{m}$ and the step size of $15 \mu \mathrm{m}$, corresponding to about 6.1 hours scan time for a $10 \times 10 \mathrm{~cm}^{2}$ patterned mask area. The illumination NA was set to 0.002 . Optical models of wave field propagation through the mask have been evaluated using rigorous coupled wave calculations to account for volumetric effects like shadowing within the absorber. The mirror was modeled 
via the transfer matrix method. The diffraction patterns were collected on a $0.33 / 4$ NA detector based on Jungfrau's design with each shot having $5 \mu \mathrm{J}$ or $3.4 \times 10^{11}$ photons arriving on the mask.

\subsection{Simulation of defect scattering}

In order to get a good overview of the expected scattering patterns and intensities from defects, simulations have been performed on a simple, ideal line pattern with $50 \mathrm{~nm}$ half pitch on mask with $5 \mathrm{~nm}$ pixel size. As a simple, perfect periodic pattern, it produced three sharp and intense diffraction peaks and with negligible intensity between the peaks. Following the simulation of the perfect pattern, we simulated a total of six different defects, including a large CD error, line gap, bridge, phase defect, small added particle and intrusion and extrusion. Moreover to evaluate potential defect impact on the printed pattern, we also generated the actinic aerial image as would be seen by the scanner. This is a rather important question as shadowing, wave guiding and reflection effects on the mask lead to the "self-healing" of certain defects, reducing their printability.

As seen on Fig. 6, each simulated defect pattern provides clearly distinct scattering pattern that can be easily distinguished from the main diffraction peaks. This allows the easy separation of defect and structure scattering without the need of any imaging optics. As will be seen in Table 1, even the smallest, barely printable defects scatter several tens of thousands photons out of the main diffraction peaks, therefore we have a considerable amount of total intensity that can be detected on the detector thus providing a large error budget for noise and measurement inaccuracies.

\subsection{Sources of noise}

The accurate evaluation of possible noise sources is of key importance towards a reliable assessment of the final sensitivity. Using the above described measurement conditions; so far we have identified three major sources of noise, of which two are coming from the measurement setup and one from the studied mask.

- Poisson noise: The photon count noise within the individual pixels may lead to poor statistics, in scanning scattering contrast microscopy thousands of pixels are summed up, eliminating the need for the evaluation of count noise within the individual pixels. Instead, it will be the count noise of the total scattered intensity that appears in our measurement. As even the smallest simulated defects are above 60000 photons at the calculated flux, the shot noise will be on the order of a few 100 photons, which is very small compared to the total signal.

- Detector noise: The average readout noise of the detector is $50 \mathrm{e}^{-}$which is equivalent to 2 photons per pixel. This is a much more significant source of measurement errors, especially for faint defects. While the large number of pixels on the detector helps to average out major fluctuations, there will always be some uncertainty left in the sum of total background with a standard deviation equivalent to $\sim 1000$ photons. As the detector readout noise is constant and independent from the incoming flux, fainter defects will show a linear dependence on the illuminating flux.

- Diffuse scattering: While large, printable defects produce strong scattering by themselves, the mask also contains a large number of small, non-printable defects in the form of surface or line-edge roughness. These defects, while not strong scatterers by themselves, are present in large numbers on any mask area and produce a diffuse scattering background with similar frequencies as printable defects. This background can be considerably higher than the scattering from fainter printable defects, but as the small defects are spread all over the mask, they represent an even background. Therefore, it will only influence the signal to noise ratio via its Poisson noise and site-to-site fluctuations. Modeling realistic roughness is a challenging task all by itself, but as the ITRS roadmap specifies $3 \sigma=2.1 \mathrm{~nm}$ absorber roughness we expect its total intensity to be on the order of $1 \mathrm{e} 7$ photons per frame. This would provide additional fluctuations similar to detector noise, but thanks to the large error budget, such fluctuations will not prevent defect recognition using suggested parameters. However it might cause problems at larger spot sizes as the ratio between the defect and background scattering scales proportional with flux density or inversely proportional to the spot area. 


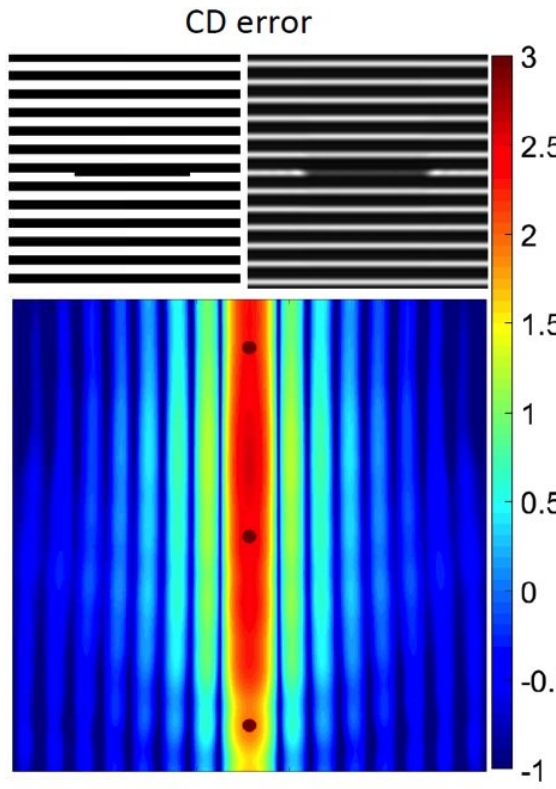

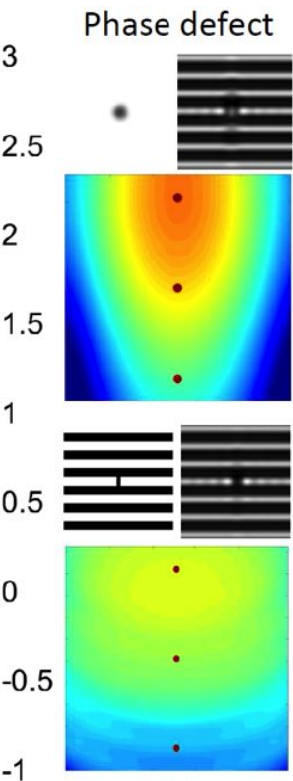

Bridge
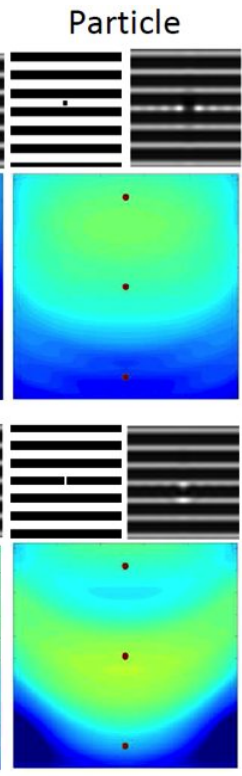

Gap

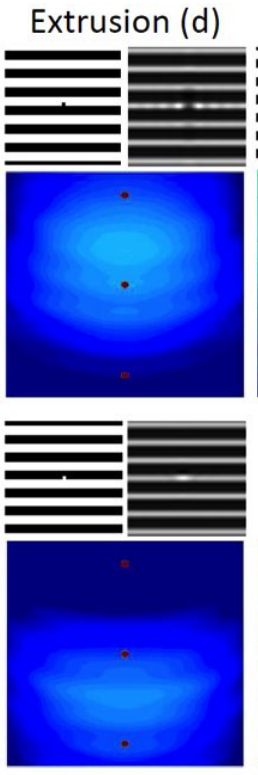

Intrusion (d)

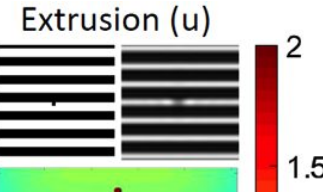

1.5

0.5

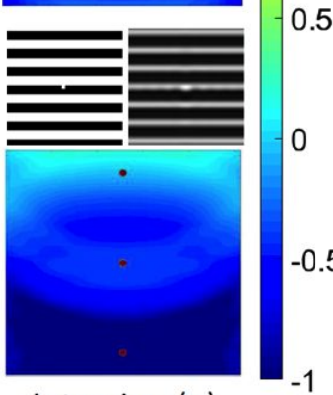

Intrusion (u)

Figure 6: Simulated mask defects, their far-field image and their calculated diffraction patterns. Whereas the CD error is $20 \mathrm{~nm} \times$ $640 \mathrm{~nm}$ (on mask) and for other defects $20 \mathrm{~nm}$ (on mask) for the smallest dimensions. The diffraction patterns are dominated by diffraction into the three main diffraction peaks as expected from defect free mask regions. However the use of logarithmic scale reveals the weak signal coming from defect scattering that is shown in units of photons per pixel. From the above images, it is clear, that defects scatter into different frequencies than regular mask structures, providing a natural separation between structure and defect scattering. As defect scattering is spread out over a large portion of the detector, it adds up to a considerable amount of total scattered intensity and is closely linked with defect printability. Besides shear defect size, also its orientation plays an important role due to shadowing.

In summary, given a source with sufficient flux, neither the Poisson noise nor the detector noise poses a significant challenge for the method. Thanks to the high flux and low measurement uncertainties, the method has plenty of margins for additional sources of noise and errors. Amongst these, roughness appears to be a notable but manageable risk if mask quality meets expectations. Therefore a quick summary of the simulated defects, their scattered intensity, and their expected sensitivity under different noise models can be seen in Table 3. For an intrusion of $20 \mathrm{~nm}$ ( $5 \mathrm{~nm}$ on wafer), which is substantially shadowed, a SNR of about 17 can be achieved. (We note that we have not evaluated the printability of the defect and have not considered the SNR increase due to multiple captures of the same defect during the scan and increased noise due to more realistic line-edge roughness values. We also didn't performed extensive optimization of measurement parameters.)

More extensive and detailed performance estimations will be studied in future work. In particular, more complex mask patterns with irregularities, assist features, etc. will be studied. The impact of multilayer roughness, and experimental errors such as positioning errors will be estimated.

Table 3: Simulated defects, their scattering intensity and their calculated signal to noise ratios for measurement errors ( $\left.\mathrm{SNR}_{\text {meas }}\right)$ and in the presence of an additional line-edge roughness of the grating $\left(\mathrm{SNR}_{\text {ler }}\right)$ with $3 \sigma=2.1 \mathrm{~nm}$.

\begin{tabular}{|l|l|l|l|l|}
\hline Defect & Size on mask (nm) & Scattered photons & $\mathbf{S N R}_{\text {meas }}$ & $\mathbf{S N R}_{\text {ler }}$ \\
\hline Perfect grating & - & 0 & 0 & 0 \\
\hline CD Error & $640 \times 20$ & $3.46 \mathrm{E}+06$ & 1183.6 & 891.0 \\
\hline Phase defect & $60 \times 60$ & $1.13 \mathrm{E}+06$ & 522.6 & 305.0 \\
\hline Bridge & $20 \times 50$ & $7.71 \mathrm{E}+05$ & 382.7 & 210.2 \\
\hline
\end{tabular}




\begin{tabular}{|l|l|l|l|l|}
\hline Gap & $20 \times 50$ & $4.93 \mathrm{E}+05$ & 260.1 & 135.3 \\
\hline DOT defect & $20 \times 20$ & $3.83 \mathrm{E}+05$ & 207.1 & 105.2 \\
\hline Extrusion (up) & $20 \times 20$ & $3.22 \mathrm{E}+05$ & 176.9 & 88.7 \\
\hline Extrusion (down) & $20 \times 20$ & $8.97 \mathrm{E}+04$ & 52.4 & 24.8 \\
\hline Intrusion (up) & $20 \times 20$ & $1.03 \mathrm{E}+05$ & 59.8 & 28.4 \\
\hline Intrusion (down) & $20 \times 20$ & $6.21 \mathrm{E}+04$ & 36.6 & 17.2 \\
\hline
\end{tabular}

\section{SUMMARY AND OUTLOOK}

In this paper we have presented a novel method for high throughput actinic EUV mask inspection, aimed to locate potential defect sites over extended areas of the mask. The method uses simple, robust algorithms based on scanning scattering. We have experimentally demonstrated the feasibility for such a setup and have provided performance estimates towards a dedicated setup. Our calculations have shown that with available source and detector technologies, large sensitivity margins with high throughput are feasible, providing plenty of headroom for the inclusion of additional uncertainties. Further short-term studies include improvement of experimental setup, in particular the optics, and simulations and experiments with more complex mask patterns. These will hopefully increase our confidence level in this method to meet the requirements of actinic mask inspection.

\section{ACKNOWLEDGEMENTS}

We would like to express our gratitude to all the members of our group at PSI and the XIL-II beamline for technical assistance and fruitful discussions. We are grateful to Albin Wrulich, M. Ehrlichmann, Thomas Schmidt, Leonid Rivkin, and Terence Garvey for their efforts and providing the information on the light source. We thank NuFlare Technology Inc., Japan, for financial support. Part of this work was performed at Swiss Light Source, Paul Scherrer Institute, Switzerland.

\section{REFERENCES}

[1] Hendrickx, E., Gronheid, R., Hermans, et al., "Readiness of EUV Lithography for Insertion into Manufacturing: The IMEC EUV Program" Journal of Photopolymer Science and Technology 26, 587-593 (2013).

[2] Amano T., Watanabe H. and Abe T., "Phase defect detection signal analysis: dependence of defect size variation" J. Micro/Nanolith. MEMS MOEMS 14(1), 013502 (2015).

[3] Hellweg D., Ruoff J., Herkommer A., Stühler J., Ihl T., Feldmann H. , Ringel M., Strößner U., Perlitz S. and Harnisch W., "AIMS ${ }^{\text {TM }}$ EUV - the actinic aerial image review platform for EUV masks" SPIE Proc, 79690H-1 (2011).

[4] Goodmann J. W., "Introduction to Fourier Optics", Roberts and Company Publishers, (2005).

[5] Juschkin L., Freiberger R. and Bergmann K., "EUV microscopy for defect inspection by dark-field mapping and zone plate zooming" Journal of Physics: Conference Series, 186, 012030 (2009) .

[6] Juschkin L., Maryasov A., Herbert S., Aretz A., Bergmann K., and Lebert R., "EUV Dark-Field Microscopy for Defect Inspection" AIP Conf. Proc., 1365, 265-268 (2011);

[7] Gerchberg R. W. and Saxton W. O., "A practical algorithm for the determination of the phase from image and diffraction plane pictures" Optik, 35, 237 (1972).

[8] Fienup J. R., "Reconstruction of an object from the modulus of its Fourier transform" Optics Letters, 3, 27 (1978).

[9] Miao J., Charalambous P., Kirz J. and Sayre D., "Extending the methodology of X-ray crystallography to allow imaging of micrometre-sized non-crystalline specimens" Nature 400, 342-344 (1999). 
[10] Rodenburg J. M., Hurst A. C., Cullis A. G., Dobson B. R., Pfeiffer F., Bunk O., David C., Jefimovs K. and Johnson I., "Hard-X-Ray Lensless Imaging of Extended Objects" Physical Review Letters, 98, 034801 (2007).

[11] Thibault P., Dierolf M., Menzel A., Bunk O., David C. and Pfeiffer F., "High-resolution scanning x-ray diffraction microscopy" Science 321, 379-382 (2008).

[12] Zheng G., Horstmeyer R. and Yang C., "Wide-field, high-resolution Fourier ptychographic microscopy" Nature Photonics, 7, 739 (2013).

[13] Harada T., Nakasuji M., Kimura T., Nagata Y., Watanabe T. and Kinoshita H., "The Coherent EUV Scatterometry Microscope for Actinic Mask Inspection and Metrology" SPIE Proc., 80810K (2011).

[14] Harada T., Nakasuji M., Kimura T., Watanabe T., Kinoshita H. and Nagata Y., "Imaging of extreme-ultraviolet mask patterns using coherent extreme-ultraviolet scatterometry microscope based on coherent diffraction imaging" J. Vac. Sci. Technol. B 29(6), 06F503-1 (2011).

[15] Harada T., Kishimoto J., Watanabe T., Kinoshita H. and Lee D. G., "Mask observation results using a coherent extreme ultraviolet scattering microscope at NewSUBARU” J. Vac. Sci. Technol. B 27 (6), 3203 (2009).

[16] Helfenstein P., Mohacsi I., Ekinci Y., "Scanning coherent diffractive imaging methods for actinic EUV mask metrology" SPIE Proc., 9776-52 (2016).

[17] Ito Y., Higuchi A., Omote K., "Characterization of cross-sectional profile of resist L/S and hole pattern using CDSAXS" SPIE proc.,9778-20 (2016).

[18] Zhang B., Gardner D. F., Seaberg M. D., Shanblatt E. R., Kapteyn H. C., Murnane M. M. and Adams D. E., "High contrast 3D imaging of surfaces near the wavelength limit using tabletop EUV ptychography" Ultramicroscopy, 158, 98 (2015).

[19] Wojdyla A. J., Benk M. P., Goldberg K. A., "Fourier Ptychography imaging for the study of EUV lithography photomasks" SPIE proc., 9776-48 (2016).

[20] Jungmann-Smith J. H., Bergamaschi A., Cartier S., Dinapoli R., Greiffenberg D., Johnson I., Maliakal D., Mezza D., Mozzanica A., Ruder Ch., Schaedler L., Schmitt B., Shi X. and Tinti G., "JUNGFRAU 0.2: prototype characterization of a gain-switching, high dynamic range imaging system for photon science at SwissFEL and synchrotrons" Journal of Instrumentation, 9, 12013 (2014).

[21] Teramoto Y., Santos B., Mertens G., Kops R., Kops M., von Wezyk A., Yabuta H., Nagano A., Shirai T., Ashizawa N., Nakamura K. and Kasama K., "High-radiance LDP source: clean, reliable, and stable EUV source for mask inspection" SPIE proc., 94220F (2016).

[22] Partlow M. J., Besen M. M., Blackborow P. A., Collins R., Gustafson D., Horne S. F., Smith D. K., "Extreme-ultraviolet light source development to enable pre-production mask inspection" J. Micro/Nanolith. MEMS MOEMS, 11(2), 021105 (2012).

[23] Miyatake T., Li X., Hirose S., Monzen T., Fujii K. and Suzuki K., "Compact synchrotron radiation lithography system for $70 \mathrm{~nm}$ device manufacturing" J. Vac. Sci. Technol. B , 19, 2444 (2001). 